\title{
Magnetic Resonance Imaging (MRI) in the Evaluation of Spinal Cord Injured Children and Adolescents
}

\author{
R. R. Betz, M.D., ${ }^{1}$ A. J. Gelman, D.O., ${ }^{2}$ G. J. DeFilipp, M.D., ${ }^{3}$ M. \\ Mesgarzadeh, M.D., ${ }^{3}$ M. Clancy, M.D., ${ }^{1}$ H. H. Steel, M.D. ${ }^{1}$ \\ ${ }_{1}$ Shriners Hospital for Crippled Children, 8400 Roosevelt Boulevard, Philadelphia, \\ Pennsylvania, U.S.A., ${ }^{2}$ Department of Orthopaedic Surgery, Albert Einstein \\ Medical Center, Philadelphia, Pennsylvania, U.S.A., ${ }^{3}$ Department of Radiology, \\ Temple University Hospital, Philadelphia, Pennsylvania, U.S.A.
}

\section{Summary}

In order to determine the indications and usefulness of MRI scanning in evaluating spinal cord trauma, MRIs on 43 subacute and chronic spinal cord injured children were compared with CT myelograms and other diagnostic tests. MRI scans were superior to CT myelograms in evaluating post-traumatic syrinx, disc pathology and the physiological status of the cord. CT myelogram remains an essential study before considering spinal cord decompression. The presence of internal fixation is not a contraindication to MRI scanning.

Key words: Magnetic resonance imaging; CT myelogram; Spinal cord injury; Children and adolescents.

\section{Introduction}

Over the past decade, medical imaging has advanced dramatically, with computerised axial tomography (CT) now used routinely in the evaluation of spinal cord trauma. Among the more intriguing of these advances has been the development of magnetic resonance imaging (MRI). This modality, which evolved from the work of Damadian (1971) and Lauterbur (1973), is being used more frequently for the diagnosis of spine problems. Modic (1983, 1984) has written on some of the uses in the spine, but the true value of MRI as a diagnostic test in spinal cord injury has yet to be established.

We report our experience with MRI in the evaluation of 43 patients with spinal cord injury. The aim of this retrospective study was to evaluate the merits of MRI in the management of spinal cord injury, and compare its value relative to plain film radiography, computerised tomography, and to myelography. 


\section{Methods}

Between June 1984 and December 1985, 43 patients with spinal cord injury were admitted to the Philadelphia Unit of Shriners Hospital. The injuries had occurred from 1 to 48 (mean $=13.4$ ) months prior to admission. The patients' ages ranged from 4 months to 21 years with a mean of 16 years. The injuries involved the cervical spine in 28 , thoracic spine in 13 , and the lumbar spine in two subjects.

The radiological examinations consisted of plain film radiography and MRI in all subjects, CT myelography in 24 , plain CT in 10 , and conventional tomography in 10 patients.

The MR studies were performed using a 0.3 Tesla permanent magnet system. In the early course of the study, the head coil was used in a few patients but later most of the patients were examined utilising the special neck surface coil. The system's resolution with the surface coil and head coil was $0.75 \mathrm{~mm}$ and 1 $\mathrm{mm}$ per pixel respectively. The slice thickness was $9 \mathrm{~mm}$ with the head coil and 2-3 mm with the surface coil. The interslice thickness was 1 or $2 \mathrm{~mm}$ with either coil. All MR studies consisted of both T1 and T2 weighted images. The pulse sequence for $\mathrm{T} 1$ weighted imaging was $\mathrm{TR}<1000 \mathrm{msec}$ and $\mathrm{TE}=28$ msec. For T2 weighted imaging TR $>1500 \mathrm{msec}$ and $\mathrm{TE}=56 \mathrm{msec}$ or longer. Sagittal images were obtained in all subjects and, in addition, axial images in 21 patients and coronal images in a few patients were available.

\section{Results}

At the onset of this review we sought to determine if MR imaging provided information equivalent to that found with CT myelography. In this study, there were $24 \mathrm{CT}$ myelograms with which to compare. In 17 cases myelograms were not performed, as it was felt that the information provided by MRI was adequate. In two cases myelograms were not indicated because no decompressive surgery was contemplated.

The MRIs correlated completely with the CT myelograms in 12 cases. In the other 12 cases differences were noted, with MRI providing additional information in nine. In the remaining three cases there was informaton on CT myelogram not seen on the MRI, but in only one case was this significant.

We specifically compared CT myelography with MRI scanniñg in assessing spinal cord compression from bone or disc after trauma. Nine patients had extrinsic cord compression (five patients from bone, two patients from disc, and two patients from both bone and disc). In comparing MRI to CT myelography specifically for evidence of compression from bone, MRI was equal in three cases, partially diagnostic in two cases, not diagnostic in one case, and was the only study to show the compression in one case. With disc compression, MRI was equal to CT myelography in two cases. In two cases compression by disc showed only on MRI scan, but surgical exploration in one of these patients did not reveal any cord compression. Repeat MRI scanning 6 months later showed the abnormal signal to be a spinal cord syrinx. This was the only false-positive scan in the study (Table).

In the cases where the MRI provided additional information, the most drama- 
Table MRI Compared with Myelogram CT

\begin{tabular}{|c|c|c|c|c|c|}
\hline & & \multicolumn{4}{|c|}{ Grade * } \\
\hline & & 0 & 1 & 2 & 3 \\
\hline \multicolumn{6}{|l|}{ Lesion } \\
\hline \multicolumn{6}{|c|}{ Extrinsic cord compression } \\
\hline \multirow[t]{2}{*}{ - Bone } & Present & 1 & 2 & 3 & $1^{(1)}$ \\
\hline & Absent & 0 & 0 & 17 & 0 \\
\hline \multirow{2}{*}{ - Disc } & Present & 0 & 0 & 2 & $2^{(2)}$ \\
\hline & Absent & 0 & 0 & 18 & 0 \\
\hline \multicolumn{6}{|c|}{ Intrinsic cord pathology } \\
\hline \multirow[t]{2}{*}{ - Syrinx } & Present & 0 & 0 & 2 & 3 \\
\hline & Absent & 0 & 0 & 19 & 0 \\
\hline \multicolumn{6}{|c|}{ - Cord atrophy } \\
\hline & Present & 0 & 0 & 1 & 6 \\
\hline & Absent & 0 & 0 & 17 & 0 \\
\hline \multicolumn{6}{|c|}{ Intervertebral disc } \\
\hline \multicolumn{6}{|c|}{ Disc abnormality } \\
\hline & Present & 0 & 0 & 1 & 12 \\
\hline & Absent & 0 & 0 & 9 & 0 \\
\hline \multicolumn{6}{|c|}{ Retropulsed disc } \\
\hline & Present & 0 & 0 & 3 & 2 \\
\hline & Absent & 0 & 0 & 19 & 0 \\
\hline
\end{tabular}

* MRI Grade: $0=$ not diagnostic; $1=$ some diagnostic information but not totally diagnostic; $2=$ as diagnostic as conventional study; 3 = additional diagnostic information provided. (1) This patient's level of injury (C7) could only be seen on MRI. His fracture-dislocation had been undiagnosed for 18 months. ${ }^{(2)}$ One of these two patients had the one false-positive result. The other patient did not have surgery, and the retropulsed disc was not confirmed.

tic finding was that of post-traumatic syrinx. Thirteen patients showed evidence on MRI of post-traumatic syrinx. Five of these patients had a CT myelogram and in only two of these were syrinxes evident (Table).

The MRI was superior for establishing the physiological status of the intervertebral disc. Twenty-three patients showed evidence of abnormal disc pathology, and of these, six had discs which retropulsed into the spinal canal (Fig. 1). Of those with retropulsed discs, five had CT myelography. Three were seen on both studies and two were seen only on MRI.

MR imaging was effective in evaluating the status of the spinal cord. MRIs demonstrated an intact spinal cord in 40 patients, two were totally unreadable due to artifact, and one patient was shown on MRI to have a totally disrupted spinal cord. Eighteen of the 40 patients with intact cords were found to have cord atrophy.

MR imaging was very helpful in establishing the level of the lesion. One 16year-old obese male presented with an incomplete C7 quadriplegia. He had been followed for 18 months elsewhere without the location of the skeletal injury ever being established. Tomograms suggested a possible malalignment of $\mathrm{C} 6$ on $\mathrm{C} 7$, but the MRI scan confirmed a complete bilateral facet dislocation of $\mathrm{C} 6$ on C7. Following confirmation of this injury, a new CT myelogram was performed and the gantry altered to the appropriate angle in order to assess the spinal cord compression, which confirmed the MRI findings. There were two patients with injuries near the cervicothoracic junction where the MRI was the only study actually confirming the level of injury. 


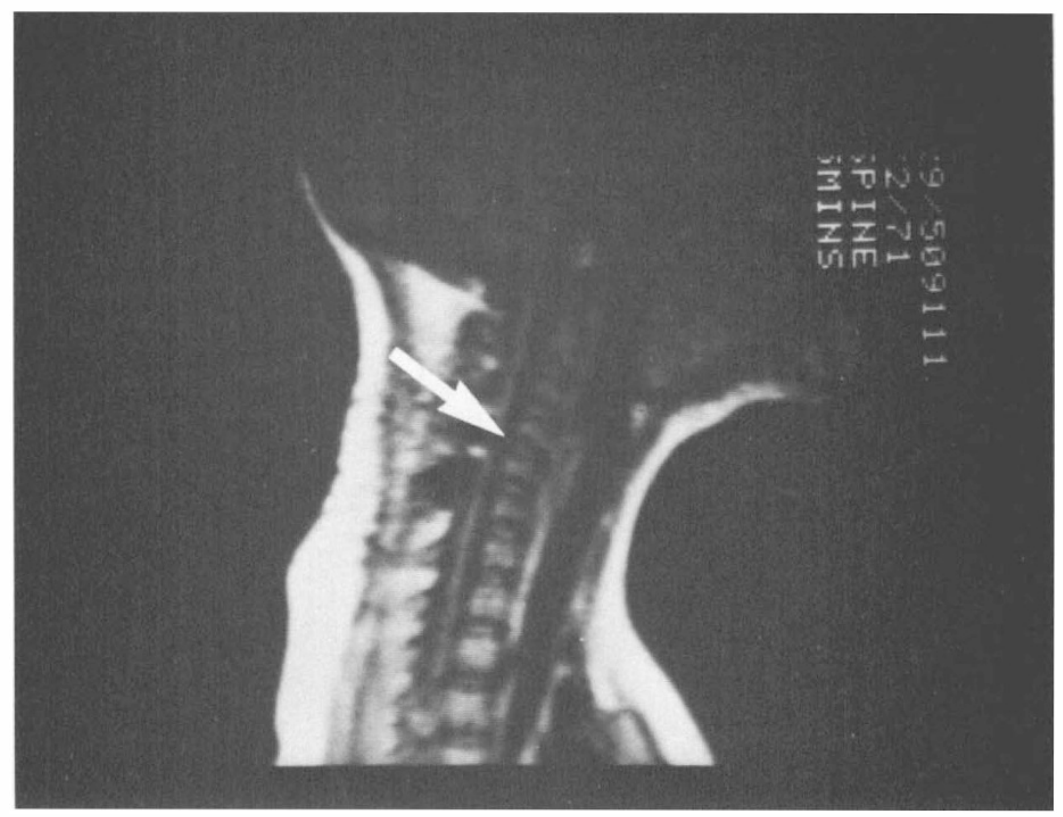

Figure 1 MRI scan of 16-year-old female 2 months status post reduction and posterior spinal fusion following bilateral facet dislocation. Note retropulsed disc causing cord compression. This was confirmed surgically and on CT myelogram.

In younger patients, the MRI was very helpful in establishing the level of the lesion when all other radiological modalities were normal. A 13-year-old patient with a $\mathrm{C} 4$ complete quadriplegia and a normal myelogram demonstrated an abnormal signal of the spinal cord at C3 on MRI. A 4-month-old infant showed a syndesmosis of $\mathrm{C} 2$ but no fracture on plain radiographs and a normal CT myelogram. The MRI scan showed an abnormal signal at C5-C6, corresponding to his clinical C6 level. One 2-year-old patient, also with normal radiographic evaluation, had an MRI which revealed a small endplate fracture and an abnormal signal on the spinal cord from $\mathrm{C} 4$ to $\mathrm{C} 7$.

There were four cases of false-negative studies where the MRI did not reveal the information seen in other studies. One patient had a vertical fracture through the vertebral body seen only on the CT scan, one patient had a foraminal entrapment visualised only on CT scan, one patient's cord compression was noted anteriorly only on the CT myelogram, and there was one patient where no anatomy at the immediate area of interest could be seen despite evidence of cord compression on the CT myelogram.

As mentioned previously, MRI revealed a false-positive result in one case. A 20-year-old patient presented with an incomplete cervical spinal cord lesion. She had a normal CT myelogram and normal metrizamide CT scan, but the MRI revealed a retropulsed disc causing extrinsic pressure on the spinal cord (Fig. 2a). After consultation with several radiologists, a neurosurgeon and two orthopaedists, it was elected to perform decompressive surgery. At the time of surgery no external compression on the spinal column was found. Postoperatively, the patient's neurological status remained unchanged, and repeat 


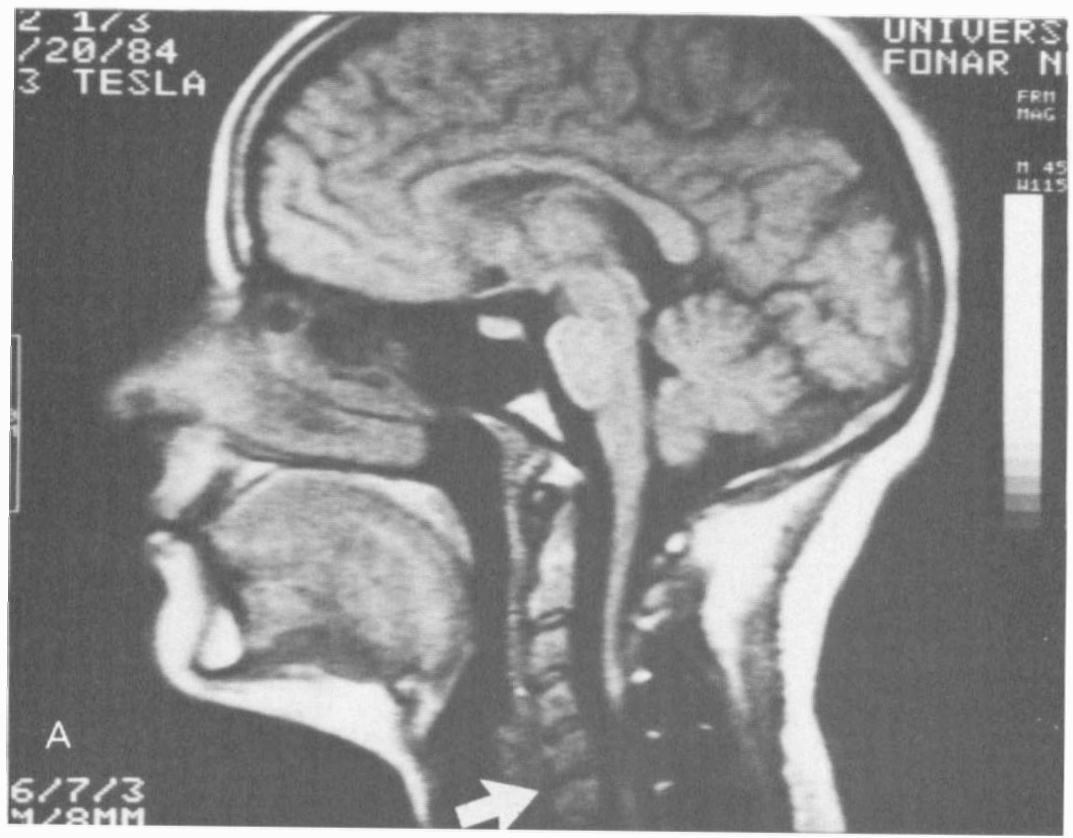

Figure 2a MRI scan of 20-year-old incomplete C5 quadriplegic showing abnormal disc (arrow) which appears to be retropulsed and compressing the spinal cord. Upon surgical exploration, no cord compression was noted. CT myelogram was normal.

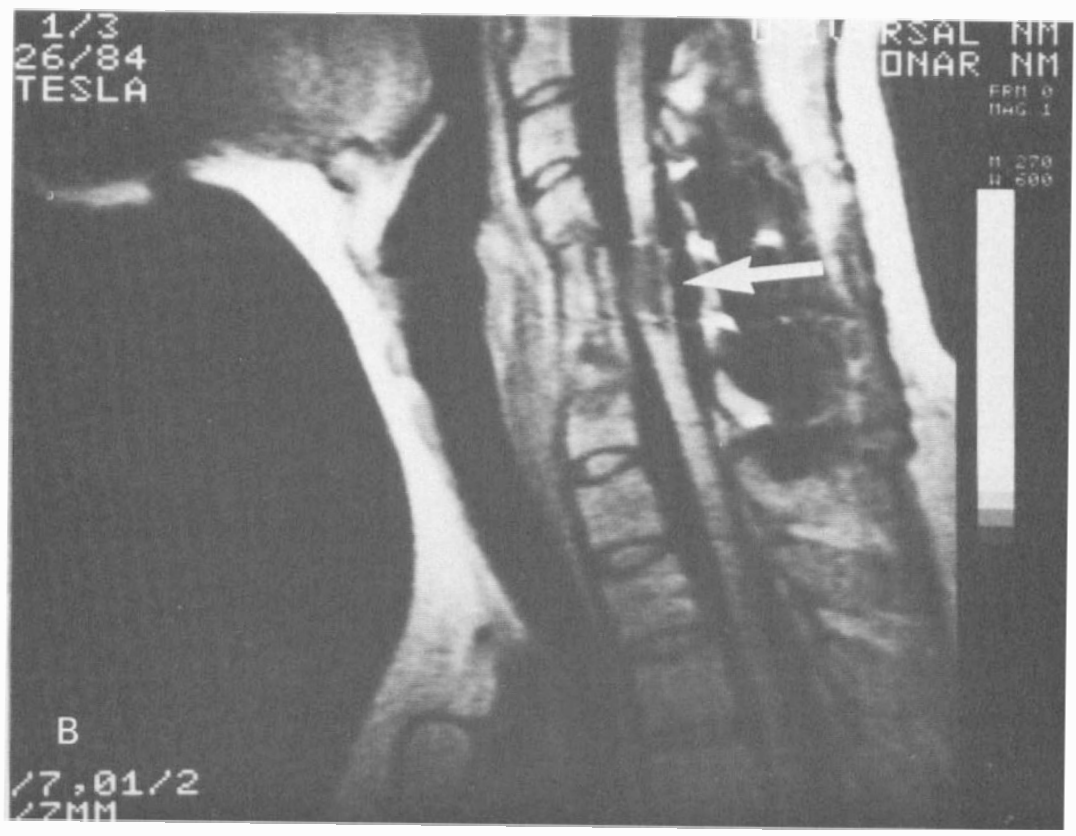

Figure 2b MRI scan of patient in Figure 2a 6 months later with new surface coil. Scan now shows spinal cord syrinx. 
scanning 6 months later with a new surface coil confirmed a spinal cord syrinx creating the original defect (Fig. 2b).

We looked next at whether metal from spine internal fixation affected MRI scanning. Twenty-three patients with internal fixation, including 17 with wires, five with Harrington rods, and one with Weiss springs, were evaluated. In only two patients was there artifact severe enough to obscure any readable information; one of these patients had Harrington rods and the other posterior wires. In four patients there was some artifact obscuring the scan, but the information obtained was still useful. In 17 patients there was absolutely no artifact due to the internal fixation, resulting in an accurate study. Two patients had bullets in the spinal column, and an accurate study was obtained in both.

\section{Discussion}

Spinal cord syrinxes appear to be one of the prime indications for the use of the MR imaging in spinal cord injury. Cystic lesions are demonstrated best in T1 weighted MRIs where the long T1 of the cyst fluid is visible compared with the intermediate strength signal from the spinal cord (Haas, 1984). Gebarski, et al., (1984) found MRI to be more specific in evaluating myelomalacia and syrinxes than delayed CT myelography in the pre-operative work-up of patients with post-traumatic ascending myelopathy. There were only two of five syrinxes in our study seen on MRI that were also seen on CT myelogram.

Our experience suggests that $\mathrm{T} 1$ images provide better anatomical resolution, which is partially due to less motion artifact as a result of short TR and therefore shorter acquisition time. However, some pathologies can be best differentiated with T2 weighted images. Cord compression and syrinx are best seen with $\mathrm{T} 1$ although they can also be demonstrated with $\mathrm{T} 2$. The degenerated discs are seen with both $\mathrm{T} 1$ and $\mathrm{T} 2$ sequences but lack of increased signal intensity in T2 helps to rule out the infectious etiology of the disc disease. T2 images are helpful in detecting intrinsic disease of the cord and demyelinisation. Therefore, we suggest that the $T 1$ weighted sagittal series is essential for MR work-up, but a TZ series would be helpful in addition.

In addition to providing a baseline for post-traumatic cyst, MRI is an excellent screen for extrinsic spinal cord compression. Also, the use of MRI eliminates the need for anti-seizure medication and the overnight hospital stay necessary for myelography.

Based on this study, we have developed the following protocol for using MR imaging in our diagnostic regimen: all patients have plain radiographs initially and then flexion-extension lateral x-rays for evaluation of instability. They next have an MRI scan to confirm the actual level of injury and to identify secondary lesions that may have not been seen in the routine studies. If compression on the spinal cord is shown on MRI and decompressive surgery may be indicated (depending on the completeness or incompleteness of the lesion or whether additional nerve roots may be gained), then a CT myelogram is ordered. In the future, with the improvement in surface coils providing better resolution, and the experience gained by physicians and technicians in conducting MRIs and correlating them clinically, we hope that CT myelograms may rarely be indicated even when considering decompressive surgery. 
Two potential problems arise with MRI scans in patients with internal fixation devices. One concern is that the magnetic fields may affect the position of the internal fixation. No evidence of this was found in any patient in our series. Recent studies by Mesgarzadeh et al., in 1985 showed that orthopaedic hip implants, including those made of stainless steel similar to spine instrumentation, did not move during MRI scanning. The second concern is that the metallic object may affect the quality of the MR image. Berquist (1984) reported artifact from both nonferromagnetic and ferromagnetic metal devices. Mechlin, et al., (1984) found no artifact with hip prostheses and surgical clips. New, et al., (1983) feels that the artifact is related to the size and degree of magnetisation. No investigation of the effect of MRI on internal fixation devices in the spine has yet been reported. In our study 17 of 23 scans were free of artifact, and in only two of 23 did the metal totally obscure the image. With improved MRI technique and specific parameters, metal artifact may be less of a problem in the future.

\section{Conclusions}

MR imaging is indicated in the evaluation of subacute and chronic spinal cord injured patients for the determination of post-traumatic syrinx, disc pathology, and the physiological status of the cord. It appears to be helpful in screening for the level of injury, spinal column alignment, and extrinsic spinal cord compression. CT myelogram remains an essential study before considering surgery for spinal cord decompression. The presence of internal fixation is not a contraindication to MRI scanning and, in the majority of cases, an accurate study is obtained.

\section{Acknowledgements}

The authors wish to express sincere appreciation to Carolyn Hill and Eileen Rosenfeld for their assistance in the preparation of this paper.

\section{References}

BERQUIST T 1984 Magnetic resonance imaging: Preliminary experience in orthopedic radiology. Magnetic Resonance Imaging 2:41-52.

Damadian R 1971 Tumor detection by nuclear magnetic resonance. Science 171:1151-1153.

GEBARSKI S, KNAKE J, GABRIELSEN T, et al.: Post-traumatic ascending myelopathy: Clinical and radiologic correlation employing $M R$ imaging, delayed CT myelography, and intraoperative ultrasonography. Abstract: p 203, Scientific Program, 70th Scientific Assembly and Annual Meeting of the RSNA, Washington, DC, Nov. 25-30, 1984.

HaAs D, Norman D, BRANT-ZawadzKi M, et al.: MR imaging of myelopathy. Abstract: p 203, Scientific Program, 70th Scientific Assembly and Annual Meeting of the RSNA, Washington, DC, Nov. 25-30, 1984.

LAUTERBUR P 1973 Image formation by induced local interactions: Examples employing nuclear magnetic resonance. Nature (London) 242:190-191.

MEsGaRzADEH M, Revesz G, Bonakdarpour A, et al. 1985 The effect on medical metal implants by magnetic fields of magnetic resonance imaging. Skeletal Radiology 14:205-206.

MODIC MT, HARDY RW, WEINSTEIN MA, et al. 1984 Nuclear magnetic resonance of the spine: Clinical potential and limitation. Neurosurgery 15:583-592.

Modic MT, Weinstein MA, PAvLICEK W, et al. 1983 Magnetic resonance imaging of the cervical spine: Technical and clinical observations. American fournal of Roentgenology 141:11291136. 
Modic MT, Weinstein MA, PAvlicek W, et al. 1983 Nuclear magnetic resonance imaging of the spine. Radiology 148:757-762.

MeChlin M, ThiCKMAN D, KRESSEL H 1984 Magnetic resonance imaging of postoperative patients with metallic implants. American fournal of Roentgenology 143:1281-1284.

New P, Rosen B, BRADY T 1983 Potential hazards and artifacts of ferromagnetic surgical and dental materials and devices in nuclear magnetic resonance imaging. Radiology 147:139-148. 